\title{
Non-parabolicity of the conduction band of wurtzite GaN
}

\author{
S. Syed, ${ }^{1}$ J. B. Héroux, ${ }^{2}$ Y. J. Wang, ${ }^{3}$ M. J. Manfra, ${ }^{4}$ R. J. Molnar, ${ }^{5}$ and H. L. Stormer ${ }^{1,4,6}$ \\ ${ }^{1}$ Department of Applied Physics and Applied Mathematics, \\ Columbia University, New York, New York 10027 \\ ${ }^{2}$ Department of Electrical Engineering, Columbia University, New York, New York 10027 \\ ${ }^{3}$ National High Magnetic Field Laboratory, Florida State University, Tallahassee, FL 32306 \\ ${ }^{4}$ Bell Laboratories, Lucent Technologies, Murray Hill, NJ 07974 \\ ${ }^{5}$ MIT Lincoln Laboratory, Lexington, MA 02420-0122 \\ ${ }^{6}$ Department of Physics, Columbia University, New York, New York 10027
}

\begin{abstract}
Using cyclotron resonance, we measure the effective mass, $m^{*}$, of electrons in AlGaN/GaN heterostructures with densities, $n_{2 D} \sim 1-6 \times 10^{12} \mathrm{~cm}^{-2}$. From our extensive data, we extrapolate a band edge mass of $(0.208 \pm 0.002) m_{e}$. By comparing our $m^{*}$ data with the results of a multi-band k.p calculation we infer that the effect of remote bands is essential in explaining the observed conduction band non-parabolicity (NP). Our calculation of polaron mass corrections - including finite width and screening - suggests those to be negligible. It implies that the behavior of $m^{*}\left(n_{2 D}\right)$ can be understood solely in terms of NP. Finally, using our NP and polaron corrections, we are able to reduce the large scatter in the published band edge mass values.
\end{abstract}

PACS numbers: 73. 20.At, 76. 40.+b, 73. 40.-c

The magnitude of the conduction band nonparabolicity (NP) in wurtzite GaN currently remains controversial. NP of a band can be probed by measuring the carrier effective mass $m^{*}$ as a function of energy. Such experiments have been performed in the past in both bulk and in two-dimensional electron systems (2DES). The deduced band edge mass values, $m_{0}{ }^{*}$, however, exhibit considerable scatter. Using cyclotron resonance (CR), Drechsler et al. 1] determined $m_{0} *$ in bulk wurtzite $\mathrm{GaN}$ to be $0.20 m_{e}$, where $m_{e}$ is the free electron mass. Other methods such as infrared reflectivity on electron plasma 2] and spectroscopy on shallow donors [3, 4, 5] in bulk GaN have yielded $0.220 m_{e}<m_{0}^{*}<$ $0.236 m_{e}$. An even wider range of values for the band edge mass, $0.185 m_{e}<m_{0}^{*}<0.231 m_{e}$, emerge from experiments in $\mathrm{AlGaN} / \mathrm{GaN}$ heterostructures. From the temperature dependence of Shubnikov-de Haas $(\mathrm{SdH})$ oscillations of 2DES, Lin et al. deduced $m_{0}{ }^{*}=0.22 m_{e}[$ [ $]$ while Hang et al. reported $m_{0}{ }^{*}=0.185 m_{e}$ [7]. Cyclotron resonance experiments on heterostructures have revealed $0.223 m_{e}<m_{0}^{*}<0.231 m_{e}$ [8, 9]. This spread in $m_{0}{ }^{*}$ suggests that the "extrapolation" from the various experiment values $m^{*}(E)$ to the band edge remains poorly controlled.

Using CR we have measured $m^{*}$ in a series of high mobility $\left(\sim 20,000 \mathrm{~cm}^{2} / \mathrm{Vsec}\right)$ AlGaN/GaN structures. Our heterostructures are all grown by molecular beam epitaxy (MBE) on GaN templates prepared by hydride vapor phase epitaxy (HVPE). The specimens are described in detail elsewhere [10, 11]. Our data cover a density range of $1-6 \times 10^{12} \mathrm{~cm}^{-2}$. In these $2 \mathrm{D}$ systems this implies energies from $\sim 27 \mathrm{meV}$ to $\sim 120 \mathrm{meV}$ above the band edge due to electron confinement and band filling. Therefore, our mass data probe the NP of the GaN conduction band in small steps over a wide energy range. We also perform extensive k.p calculations and determine that instead of the commonly used two-band model, a multi-band model is required to explain our experimental results. Additionally, our calculations on polaron correction of the effective mass of $2 \mathrm{D}$ electrons in GaN show them to be at the $1 \%$ level, considerably less than previously thought [8]. A detailed comparison between our data and k.p calculation sets the band mass value to $m_{0}^{*}=(0.208 \pm 0.002) m_{e}$ and, when applied to other investigators' results, considerably reduces the spread in band edge mass values.

A Fourier transform spectrometer with light pipe optics and a composite Si bolometer was used for the detection of far-infrared transmission. Magnetic field was applied normal to the 2D electron layer. The carrier density of each sample was determined in situ from the Shubnikov-de Haas (SdH) oscillations of the 2DES. All $\mathrm{CR}$ and $\mathrm{SdH}$ experiments were conducted at $4.2 \mathrm{~K}$.

Fig 1 shows the cyclotron resonance energies versus magnetic field, $B$ of a sample with $n_{2 D}=2.3 \times 10^{12} \mathrm{~cm}^{-2}$. All data are taken with a resolution of $0.24 \mathrm{meV}$. The inset shows high field transmission spectra normalized to the spectrum taken at $B=0 \mathrm{~T}$. The solid line in Fig.1 is a fit to the data at high fields, $B>27 \mathrm{~T}$, and low fields, $B<12 \mathrm{~T}$, resulting in an effective mass of $m^{*}=0.228 m_{e}$. For $15 \mathrm{~T}<B<25 \mathrm{~T}$, there is a pronounced deviation from this straight line. This anomaly in the CR represents a recent discovery, which is being analyzed and published elsewhere [10]. Here, we observe that this anomaly is limited to a finite field region outside which all $\mathrm{CR}$ data can be fit by a straight line.

We have measured the effective mass in eleven samples with carrier density, $n_{2 D}$, ranging from $1-6 \times 10^{12} \mathrm{~cm}^{-2}$. In all cases, we observed either a broadening or a splitting of the CR line at intermediate fields but could fit the data away from this regime as well as the data seen in Fig. 1] 


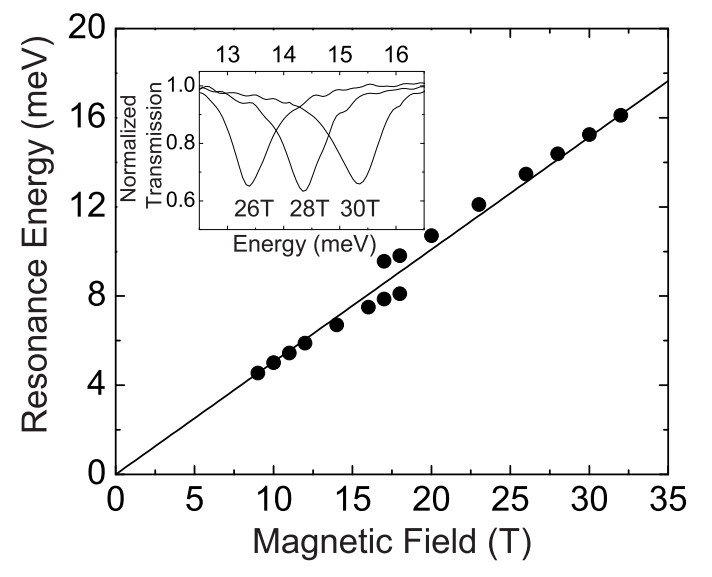

FIG. 1: Resonance energies vs. $B$ of a sample with $n_{2 D}=$ $2.3 \times 10^{12} \mathrm{~cm}^{-2}$. High and low field resonances can be fit with a single straight line, giving an effective mass of $0.228 m_{e}$. Near $18 \mathrm{~T}$ a level anti-crossing results in a splitting of the CR (see text). Inset: Transmission data for $B=26,28$, and $30 \mathrm{~T}$, normalized to the spectrum at $B=0 \mathrm{~T}$.

Fig[2] shows the dependence of $m^{*}$ on $n_{2 D}$. For comparison, we also plot data from Ref. [8, 9, 12]. The mass data from the different references are located in the general vicinity of our results but, due to their considerable error bar or sparsity, are difficult to extrapolate to zero density. The combined data of Fig 2 show an increase in $m^{*}$ by $\sim 17 \%$ as $n_{2 D}$ changes from $1-9 \times 10^{12} \mathrm{~cm}^{-2}$. The rise in $m^{*}$ with $n_{2 D}$ reflects the non-parabolicity of the conduction band of the GaN host. Simply extrapolating our closely spaced data linearly to vanishing $n_{2 D}$, we arrive at $m_{0}^{*}=0.214 m_{e}$. This value is about $\sim 8 \%$ lower than previously published CR data [8, 9]. Since our data contain a small error bar and extend to very low $n_{2 D}$, such a simple extrapolation should already be quite reliable.

Other groups have previously addressed NP in GaN. For example, Knap et al. explored NP using CR with different $n_{2 D}$ (see our Fig 2) and accounted for the magnitude of NP using a simple two-band approximation [8, 9]. In such an approximation, which includes only coupling between the lowest conduction and highest valence bands, the effective mass varies as

$$
m^{*}(E)=m_{0}^{*}\left(1+2 K E / E_{g}\right)
$$

with $K=1$ and $E_{g}=3.5 \mathrm{eV}$. In a $2 \mathrm{DES}$ the energy, $E=$ $E_{k}+E_{F}$, above the band minimum is composed of the average kinetic energy, $E_{k}$, of the electrons in the confining potential well and $E_{F}=\pi \hbar^{2} n_{2 D} / m_{0}^{*}$ [13, the Fermi energy of the $2 \mathrm{D}$ electron system. The value of $E_{k}$ is dependent on the form of the wave function of the confined electrons. In a simple triangular potential approximation, the average kinetic energy is $E_{k}=E_{c} / 3$, with $E_{c}$ being the confinement energy of the lowest subband [14]. Using the more accurate Fang-Howard

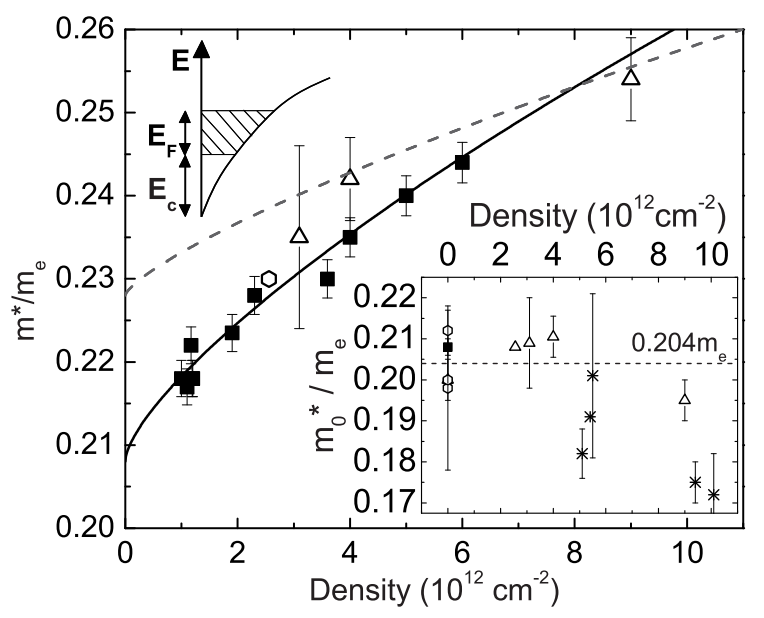

FIG. 2: Effective mass, $m^{*}$, vs. 2D electron density. Our data are shown as solid squares. Data from Ref. [8, 9] and 12] are shown as open symbols. The dashed line is a two band fit to the results of Ref. [9] according to Eq11with $K=1$. The solid line represents a fit with $K=2.5$, which accounts for the influence of additional, higher conduction bands. Inset: Values of the band edge mass from Refs. 1, 2, 3, 4, 5, 6, 7, 8, 9, 12] and this work, after NP and polaron corrections. CR results are shown as open triangles, donor spectroscopy data as open hexagons and $\mathrm{SdH}$ data as stars. Our result of $m_{0}^{*}=(0.208 \pm 0.002) m_{e}$ is shown as a solid square. Averaging all data (except $\mathrm{SdH}$ ) yields $m_{0}^{*}=0.204 m_{e}$ illustrated by a dashed line (see text).

variational wave function gives $E_{k}=\hbar^{2} b^{2} / 8 m_{0}^{*}$, where $b^{3}=48 \pi m_{0}^{*} e^{2}\left(N_{\text {dep }}+\frac{11}{32} n_{2 D}\right) / \epsilon \hbar^{2}[14]$. In our samples, since both the MBE and the HVPE GaN are $n$-type, the depletion layer density, $N_{d e p}$, can be set to zero. We use this two-band model with only one adjustable parameter, $m_{0}^{*}$, to fit the high-density data of Ref. [9] (dashed line in Fig (2). $E_{k}$ was computed in the triangular approximation following the authors of Ref. [9]. Clearly, although this procedure can describe the original density dependence of $m^{*}$ of Ref. [9] due to the rather large error bars, it fails to account for our data. Neither a simple vertical shift of the line (a different $m_{0}^{*}$ ) nor the usage of the Fang-Howard wavefunction can resolve this discrepancy. What is required, is a much stronger dependence of $m^{*}$ on $n_{2 D}$.

Empirically, we pursue an approach taken by Singleton et al. [15], who used a modified two-band model to describe their NP data in GaAs. The authors considered $K$ in Eq⿴ to be a second fitting parameter. The inclusion of a variable $K>1$ into the analytic expression incorporates the influence of higher conduction bands, simulating the results of a more elaborate, multi-band k.p calculation 16]. Working with the Fang-Howard model to determine $E_{k}$ we find a very good fit to our data for $K=2.5$ and $m_{0}^{*}=(0.208 \pm 0.002) m_{e}$. Even if we use the less reliable triangular approximation, the required $K=$ 1.9. The effectiveness of the modified expression in fitting 
the density dependence of $m^{*}$ over a wide range of $n_{2 D}$ demonstrates that the conduction band of $\mathrm{GaN}$ is more non-parabolic than was previously assumed [6, 7, 8, 9].

Before concluding that the proposed NP model appropriately describes our CR data, we need to assure ourselves that polaron effects are a negligible contributor to $m^{*}$. This mass enhancement factor results from electronLO phonon coupling in polar semiconductors, such as GaN. A determination of a polaron mass enhancement in heterostructures requires inclusion of screening in 2D and the finite width of the electronic wave function [17], since both greatly reduce interaction between $2 \mathrm{D}$ carriers and LO phonons [18]. Following Ref. [18], we calculated the polaron effective mass in AlGaN/GaN heterostructures using a a Frohlich constant, $\alpha=0.49$ [1], FangHoward variational wave function, and a static ThomasFermi screening model. We find a polaron enhancement of less than $1 \%$ for $m^{*}$ for $n_{2 D}=9 \times 10^{12} \mathrm{~cm}^{-2}$. Since the effect decreases with decreasing density, corrections for lower density specimens are smaller yet. This mass enhancement is considerably smaller than the $10 \%$ estimated previously for $n_{2 D}=3.1 \times 10^{12} \mathrm{~cm}^{-2}$ [8], where screening and finite width had been neglected. A $1 \%$ mass enhancement due to polaronic coupling lies within the error bars of our CR data.

The large spread in the value of the band edge mass $m_{0}^{*}$ in the literature is mostly due to two reasons: the underestimation of the NP and the overestimation of polaronic corrections. Applying our NP and polaron corrections to the available effective mass data (Ref. 1, 2, 3, 4, 5, 6, 7, 8, 9, 12]), we reach a much more coherent picture for the band edge effective mass, $m_{0}^{*}$, in GaN, as shown in the inset to Fig. 22 We observe that the majority of the values for $m_{0}^{*}$ are very close to an average $m_{0}^{*}=0.204 m_{e}$. However, most of the $m_{0}^{*}$ data from $\mathrm{SdH}$ (displayed in stars) remain at variance from the CR, infra-red reflectivity and donor spectroscopy data, a fact that remains unexplained.

Since $m^{*}(E)$ cannot be accurately represented with a two-band model, a five-band k.p calculation 16] in the zincblende approximation was performed to model our data. Figure 3 shows the results. The material parameters employed using the Koster notation are the spinorbit splitting, $\Delta_{0}^{\prime}$, of the $\Gamma_{5}$ conduction band and the momentum matrix elements, $P^{2}$ and $\lambda^{2} P^{2}$, coupling the $\Gamma_{1}$ conduction band with the $\Gamma_{5}$ valence and conduction bands. They are not precisely known. The formalism proposed by Carlos et al. [19] and used by Bayerl et al. [20] in a 5-band model to relate the parameters was utilized to calculate $m^{*}(E)$. However, as seen in Fig 3 the parameters chosen by Bayerl et al. lead to a non-parabolicity even lower than in a two-level model. We find that the experimental data points can only be matched if an extra parameter $C$, taking remote bands into account at $k=0$, is included 21]. If one assumes $C=-1.5$ (compare with $C=-2$ for GaAs [16]), $P^{2}$ is in

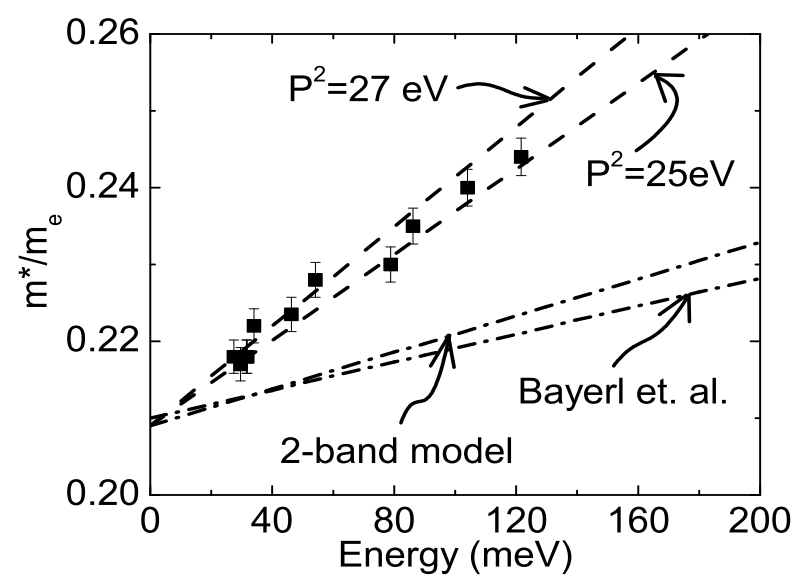

FIG. 3: Variation of $m^{*}$ with energy according to k.p calclations, assuming $m_{0}^{*}=0.208 m_{e}$. The two-band model and the five-band k.p results of Bayerl et al., which neglect remote bands both underestimate the energy-dependence of $m^{*}$. The experimental data (solid squares) can be explained by multiband k.p calculations that include the influence of remote bands (dashed lines).

the range $25-27 \mathrm{eV}$ with $\lambda^{2} \approx 0.33-0.48$ and $\Delta_{0}^{\prime} \approx 120-180$ $\mathrm{meV}$. This set of parameters is close to the "best" set of values chosen by Kennedy et al. 22] and confirm that $P^{2}$ is relatively large for $\mathrm{GaN}\left(P^{2} \approx 26 \mathrm{eV}\right)$.

In conclusion, our $\mathrm{CR}$ experiments set the conduction band edge mass in GaN to $m_{0}^{*}=(0.208 \pm 0.002) m_{e}$. Using our determination of the nonparabolicity and reviewing the polaron correction we reach much better agreement between several published data for $m_{0}^{*}$.

We thank M.S. Brandt, L.N. Pfeiffer, K.W. West, and W.I. Wang for helpful discussions. A portion of the work was performed at the National High Magnetic Field Laboratory, which is supported by NSF Cooperative Agreement No.DMR-0084173 and by the State of Florida. Financial support from the W. M. Keck Foundation is gratefully acknowledged.

[1] M. Drechsler et al., Jpn. J Appl. Phys. 34, L1178 (1995).

[2] P. Perlin et al., Appl. Phys. Lett. 68, 1114 (1996).

[3] W. J. Moore et al., Phys. Rev. B 56, 12073 (1997).

[4] A. M. Witowskia et al., Appl. Phys. Lett. 75, 4154 (1999).

[5] B. K. Meyer et al., Solid State Comm. 95, 597 (1995).

[6] T. Y. Lin et al., Phys. Rev. B 58, 13793 (1998).

[7] D. R. Hang et al., Appl. Phys. Lett. 79, 66 (2001).

[8] W. Knap et al., Solid State Commun. 99, 195 (1996).

[9] W. Knap et al., 70, 2123 (1997).

[10] S. Syed et al., Phys. Rev. B 67, 241304 (2003).

[11] M. J. Manfra et al., J. Appl. Phys 92, 338 (2002).

[12] Y. J. Wang et al., J. Appl. Phys. 79, 8007 (1996).

[13] Here we use an average mass of $m_{0}^{*}=0.23 m_{e}$, since it enters $E_{F}$ as $\left(m_{0}^{*}\right)^{-1}$ and $E_{k}$ as $\left(m_{0}^{*}\right)^{-1 / 3}$ and therefore 
enters $m^{*}$ in higher order.

[14] T. Ando et al., Rev. Mod. Phys. 54, 437 (1982).

[15] J. Singleton et al., Surf. Sci. 196, 429 (1988).

[16] P. Pfeffer and W. Zawadzki, Phys. Rev. B 41, 1561 (1990).

[17] According to Ref. [18] phase-space restrictions due to the degeneracy of the 2DES can be ignored for $k_{F} / q_{0}$ at least as high as 0.6 , where $q_{0}$ is the phonon wavevector and $k_{F}$ the Fermi wavevector. In our $\mathrm{AlGaN} / \mathrm{GaN}$ systems $k_{F} / q_{0}$ $\leq 0.6$ for $n_{2 D} \leq 3.4 \times 10^{12} \mathrm{~cm}^{-2}$ and still $k_{F} / q_{0} \approx 0.8$ for our highest density. We therefore also ignore phase space restriction.

[18] S. DasSarma, Phys. Rev. B 27, 2590 (1983).

[19] W. E. Carlos et al., Phys. Rev. B 48, 17878 (1993).

[20] M. W. Bayerl et al., Phys. Rev. B 63, 165204 (2001).

[21] C. Hermann and C. Weisbuch, Phys. Rev. B 15, 823 (1977).

[22] T. A. Kennedy et al., MRS Internet J. Nitride Semicond. Res. 4S1, G7.4 (1999). 